\title{
523676 (2013 UL10): the first active red centaur ${ }^{\star}$
}

\author{
E. Mazzotta Epifani ${ }^{1}$, E. Dotto ${ }^{1}$, S. Ieva ${ }^{1}$, D. Perna ${ }^{1,2}$, P. Palumbo $^{3,4}$, M. Micheli ${ }^{5,1}$, and E. Perozzi ${ }^{6}$ \\ ${ }^{1}$ INAF - Osservatorio Astronomico di Roma, Via Frascati 33, 00078 Monte Porzio Catone, Roma, Italy \\ e-mail: elena.mazzottaepifani@inaf.it \\ 2 LESIA - Observatoire de Paris, PSL Research University, CNRS, Sorbonne Universités, UPMC University Paris 06, University \\ Paris Diderot, Sorbonne Paris Cité, 5 place J. Janssen, 92195 Meudon, France \\ ${ }^{3}$ Dipartimento di Scienze e Tecnologie, Universitá Parthenope, Centro Direzionale Isola C4, 80143 Napoli, Italy \\ ${ }^{4}$ INAF - IAPS, Via Fosso del Cavaliere 100, 00133 Roma, Italy \\ ${ }^{5}$ ESA SSA-NEO Coordination Centre, largo Galileo Galilei 1, 00044 Frascati, Roma, Italy \\ ${ }^{6}$ ASI-Agenzia Spaziale Italiana, Roma, Italy
}

Received 23 May 2017 / Accepted 21 September 2018

\begin{abstract}
Aims. We present observations of 523676 (2013 UL10), a centaur orbiting between Jupiter and Uranus that is dynamically similar to the few tens of active centaurs that are currently known.

Methods. We analysed visible BVR images of the centaur obtained at the Telescopio Nazionale Galileo (La Palma, Canary Islands, Spain) to investigate the weak comet-like activity and to derive information on the nucleus surface colours and size.

Results. Centaur 523676 (2013 UL10) is the only centaur known so far that has both comet-like activity and red surface colours: its nucleus has a colour index $[B-R]=1.88 \pm 0.11$. The nucleus $R$ magnitude $(R=20.93 \pm 0.09)$ allowed us to derive an upper limit for its nucleus size of $D \leq 10 \mathrm{~km}$. We estimated its dust production rate to be $Q_{\mathrm{d}} \sim 10 \mathrm{~kg} \mathrm{~s}^{-1}$ at 6.2 au (just after its perihelion passage), resulting in a timescale for the surface blanketing process $\tau_{B}$ of approximately tens of years, which is very short with respect to typical dynamical lifetime inside the group. Future monitoring of 523676 (2013 UL10) is needed to further constrain the blanketing model for active centaurs and its timescale.
\end{abstract}

Key words. minor planets, asteroids: individual: 523676 (2013 UL10)

\section{Introduction}

Centaurs form a dynamical class of small bodies in the solar system that move on highly chaotic and unstable orbits in the region between the orbits of Jupiter and Neptune. With orbital lifetimes of the order of $10^{6} \mathrm{yr}$ (Tiscareno \& Malhotra 2003), centaurs are briefly residents in the region between the gas giant planets, and those who survive the dynamical environment in this region may become Jupiter-family comets (JFCs; Levison \& Duncan 1992; Horner et al. 2004). As they are considered objects in transition from the inactive Kuiper belt objects to the active JFCs, the study of their physical properties is a main topic for assessing the relationship and establish reliable patterns between the object classes, and to constrain the evolution of minor bodies in the solar system.

A few centaurs ( 30 objects in a family of $\sim 300$ members, i.e., around $10 \%$ of the whole sample, as of January 2018$)^{1}$ have been observed to have a well-developed dust coma in optical images. The first example was (2060) Chiron, which orbits at about $10 \mathrm{au}$ from the Sun and shows a sustained but variable cometary-like activity along its orbit (Luu \& Jewitt 1990; Meech \& Belton 1990). The activity of centaurs is part of a wider debate on the activity of small bodies at great distance from the Sun beyond

\footnotetext{
${ }^{\star}$ Based on observations collected at the Telescopio Nazionale Galileo (TNG), operated on the island of La Palma by the Centro Galileo Galilei of the INAF (Istituto Nazionale di Astrofisica) at the Spanish Observatorio del Roque de los Muchachos of the Instituto de Astrofísica de Canarias.

1 https://physics.ucf.edu/ yfernandez/cometlist.html
}

the so-called water zone (i.e., the region with temperatures that enable water-ice direct sublimation). Beyond 5-6 au, comet-like activity cannot be explained with classical water-ice direct sublimation (Meech \& Svoren 2004). Models of cometary nuclei (e.g. Prialnik 1997; Capria et al. 2000a,b) and laboratory experiments (e.g. Notesco et al. 2003) showed that amorphous water ice is highly porous and can contain very many (hyper)volatile molecules that were trapped at the time of condensation. The trapped gas could be released upon ice crystallisation, giving rise to dust loss at temperatures that are too low (and hence at heliocentric distances too large) for crystalline water ice to sublimate. Figure 1 shows the distribution of the orbital eccentricity of all known centaurs versus the semi-major axis. It is quite interesting to observe that all the centaurs that were firmly identified to have a comet-like appearance (and in some case also with a cometary designation) fall in (or very close to) the region delimited by the loci of orbits whose perihelia are equal to semi-major axes of Jupiter and Saturn (as preliminary introduced by Jewitt 2009). We use a working definition of a potential activity region here for this zone. The inner edge of this region is a fictitious curve that we can draw to dynamically separate the centaurs from the JFCs, and Jupiter's distance is not related with activity, as evidenced by the fact that the JFCs inside that line are active. The interesting distance is the apparent outer edge of the active region.

Comet-like activity in this potential activity region might be due in large part to observational bias. Jewitt (2009) discussed the issue of comet-like activity of centaurs in terms of the phase transition of amorphous ice and following release of trapped gases upon crystallisation. The authors showed that in a 


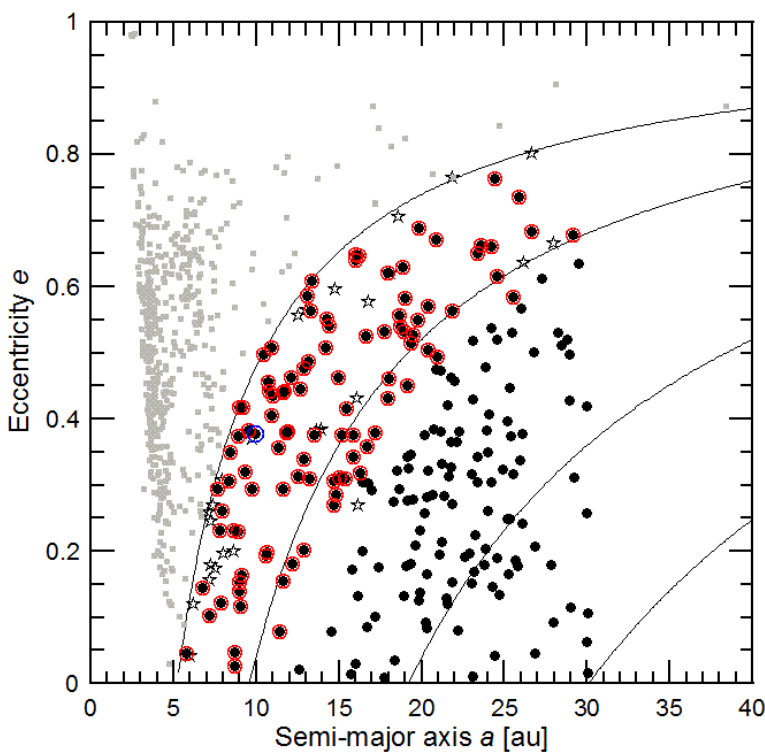

Fig. 1. Orbital semi-major axis vs. eccentricity for inactive (black dots) and active (stars) centaurs (as of July 2017). Continuous curves show the loci of orbits whose perihelia are equal to the semi-major axes of the four giant planets. Jupiter-family comets (small grey dots) are also included in the plot. Among inactive centaurs, the red-circled black dots show centaurs in the potential activity region (see text). The blue-circled black dot is Centaur 523676 (2013 UL10).

simplified model of ice crystallisation on a timescale comparable to a centaur's orbit, solving the energy balance equation for the two extreme cases of temperature (subsolar point and isothermal blackbody approximation) would lead to a critical distance inside which exposed ice must be crystalline, and therefore the phase transition is expected at $6.8 \leq r_{\mathrm{h}} \leq 14.0$ au.

Nevertheless, the real fraction of active centaurs is unclear, as is why more than half of the centaurs that might be comets are inactive instead, and most of all, how and to which extent the physical studies of centaurs are stymied by a possibly underestimated coma contribution. Very few active centaurs have been fully characterised up to now: the dust loss rate $Q_{\mathrm{d}}$ for many of them is comparable or even higher than the rate of several active comets at much smaller heliocentric distances (Mazzotta Epifani et al. 2006, 2011; Jewitt 2009; Lacerda 2013). On the other hand, few active centaurs can be considered quite weak dust emitters, despite their relatively close perihelion distance, with $Q_{\mathrm{d}}$ on the order of $\sim 10 \mathrm{~kg} \mathrm{~s}^{-1}$ (Jewitt 2009; Mazzotta Epifani et al. 2014, 2017; Kulyk et al. 2016).

Visual colours of centaurs exhibit (from the very first studies) a peculiar distribution: they apparently divide the population into two distinct groups, grey centaurs (with neutral, solar-like colours), and red centaurs (with very red colours; e.g. Tegler \& Romanishin 2003, Peixinho et al. 2003, Dotto et al. 2003). The colours of this class of objects are crucial to obtain reliable hints on the surface properties and their evolution within the solar system: it is still unclear if the peculiar colour distribution of centaurs is due to different thermal reprocessing on their surface (Melita \& Licandro 2012; Perna et al. 2013) or to a different composition and/or region of origin (Peixinho et al. 2012; Fraser \& Brown 2012).

As a group, active centaurs were up to very recently clustered in the grey group, and their mean colours overlapped with those of the JFC nuclei. Only recently (Jewitt 2015), a few active centaurs were found for the first time with colours that classified them as intermediate among the two groups, and Tegler et al.
Table 1. Orbital elements of centaur 523676 (2013 UL10) (from JPL Horizon Database).

\begin{tabular}{ccccc}
\hline \hline$e^{a}$ & $a^{b}(\mathrm{au})$ & $q^{c}(\mathrm{au})$ & $i^{d}\left({ }^{\circ}\right)$ & $P^{e}(\mathrm{yr})$ \\
\hline 0.378 & 9.97 & 6.20 & 19.15 & 31.5 \\
\hline
\end{tabular}

Notes. ${ }^{(a)}$ Eccentricity; ${ }^{(b)}$ semi-major axis; ${ }^{(c)}$ perihelion distance; ${ }^{(d)}$ inclination; ${ }^{(e)}$ orbital period.

(2016) also found a few inactive centaurs with colours tending to reduce the gap.

The question of centaur colours and their relationship with comet-like activity is still open: one hypothesis is that the primordial surface of bodies coming from the Kuiper belt, consisting of irradiated organics spread on a more or less thick surface layer, is progressively blanketed with fresh, un-irradiated material expelled from below after the rise of comet-like activity. Blanketing timescales are quite uncertain, since centaur activity could be episodic and fallback will not, in general, be uniformly distributed on the nucleus surface, but some observational evidence allows us to estimate that it could be very short $(0.1-10 \mathrm{yr}$; Jewitt 2015) compared to the typical dynamical lifetime $\left(10^{6} \mathrm{yr}\right)$ of a centaur.

In November 2015, our group started a pilot program to collect broadband visible images of several members of the inactive centaurs class, in order to investigate their surface colours and taxa and the level of a possible coma contamination in centaurs within the potential activity region. The analysis of results is ongoing. The aim of this paper is to report on our observations of centaur 523676 (2013 UL10), which is flagged as inactive and within the potential activity region (see Fig. 1). The current orbital elements of the centaur are reported in Table 1. This object was observed by Tegler et al. (2016): reddish colours were found, while the possible presence of a cometary-like activity was not investigated. According our observations, this centaur is the only centaur currently known with both comet-like activity (very likely just after its onset) and red colours of its nucleus surface. Sect. 2 of this paper describes the observations and the data reduction. In Sect. 3, we describe the observational results, while in Sect. 4 we discuss the peculiar colour properties of 523676 (2013 UL10), observed for the first time in the centaur class, their implications, and some future perspectives.

\section{Observations and data reduction}

TNG images of 523676 (2013 UL10) were obtained during the night of 11-12 December 2015 with the Device Optimised for the LOw RESolution (DOLORES) instrument. The detector is a $2048 \times 2048 \mathrm{E} 2 \mathrm{~V} 4240$ thinned back-illuminated, deepdepleted, Astro-BB coated CCD with a pixel size of $13.5 \mu \mathrm{m}$. The scale is $0.252 \mathrm{arcsec} / \mathrm{px}$. The instrument was equipped with the broadband filters $B, V$, and $R$ of the Johnson-Cousins system $^{2}$. The circumstances of observations are summarised in Table 2. The observing conditions were quite good and the seeing was quite stable during the observations, with a mean value of $\sim 1 \operatorname{arcsec}(F W H M)$ in the $R$ filter. With the telescope tracking at the nonsidereal motion rate of 523676 (2013 UL10), seven consecutive images have been acquired (exposure time of $300 \mathrm{~s}$ each), following the sequence RVBRVBR, in the airmass range 1.4778-1.2753. The images were reduced using standard procedures (subtraction of masterbias and flat-field correction),

2 http://www.tng.iac.es/instruments/filters/ 
Table 2. Circumstances of observation of centaur 523676 (2013 UL10) at TNG.

\begin{tabular}{ccccc}
\hline Date $^{a}$ & $r^{b}(\mathrm{au})$ & $\Delta^{c}(\mathrm{au})$ & $\alpha^{d}\left({ }^{\circ}\right)$ & $\mathrm{PA}^{e}\left({ }^{\circ}\right)$ \\
\hline 11 December 2015 23:45 UT & 6.62 & 5.73 & 3.97 & 307.9 \\
\hline
\end{tabular}

Notes. ${ }^{(a)}$ Beginning of the image sequence (see text); ${ }^{(b)}$ heliocentric distance; ${ }^{(c)}$ geocentric distance; ${ }^{(d)}$ phase angle; ${ }^{\left({ }^{(}\right)}$position angle of the extended Sun-target radius vector as seen in the observer's plane of sky, measured counter-clock wise.

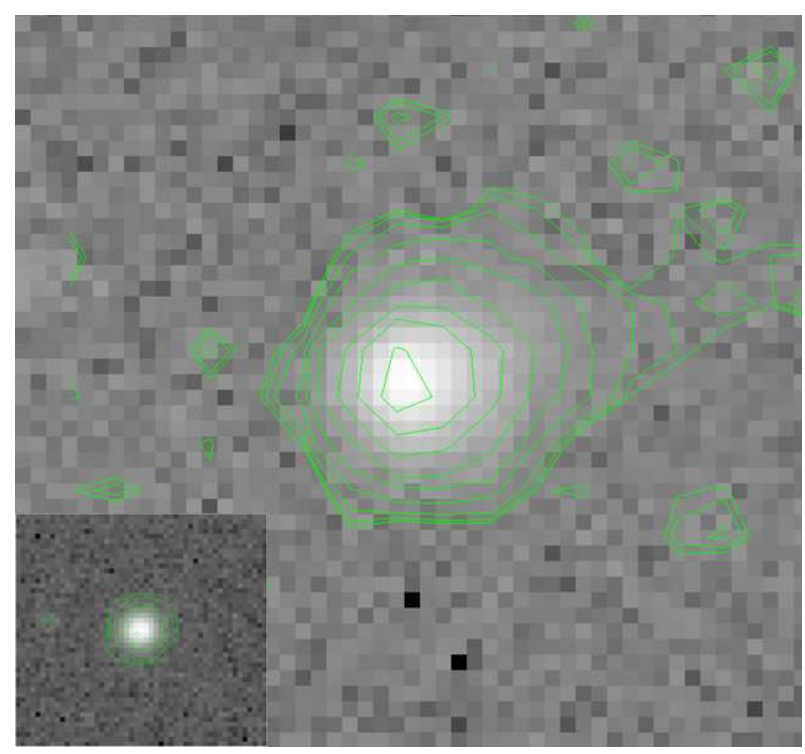

Fig. 2. Final stacked image of centaur 523676 (2013 UL10), taken on 11 December 2015. The image is in the $R$ filter, and is the coadding of three individual frames for a total exposure time of $900 \mathrm{~s}$. Median airmass is 1.35 . The image is displayed in log scale to enhance the lowlevel activity, together with overplotted isophotes. The linear scale of the image is $5.3 \times 10^{4} \mathrm{~km}$. North is up and east is to the left. In the lower left corner, the stacked image of inactive centaur 2008 FC76 (together with overplotted isophotes) obtained in the same observing night is reported for comparison (see text).

by means of the ESO Munich Image Data Analysis System (MIDAS 1998). For each filter, the individual reduced images were recentred on the centaur optocentre (found by fitting a 2D Gaussian to the target) and then summed, in order to increase the signal-to-noise ratio for the image analysis. Sky correction was then performed by subtracting a sky-annulus around the centaur (outside a diameter of $10^{\prime \prime}$ centred on the optocentre) in the final stacked image for each filter. As an example for the images obtained in all filters, the $R$ final stacked image is shown in Fig. 2.

The absolute photometric calibration was performed by computing the photometric zero-points and extinction coefficients for each filter by a least-square fit for many standard fields (Landolt 1992) taken at different airmass values during the observing night.

\section{Results}

\subsection{Comet-like activity}

Each raw $R$ image of 523676 (2013 UL10) showed even at a first sight clear hints of comet-like activity. This was even more evident when the pre-reduced frames were summed to obtain the final stacked image. Asymmetric (with respect to the optocentre)
Table 3. Magnitude and colours of centaur 523676 (2013 UL10) in selected apertures centred on the centaur optocentre.

\begin{tabular}{ccccc}
\hline \hline$\rho^{a}\left({ }^{\prime \prime}\right)$ & $R$ & $B-V$ & $V-R$ & $B-R$ \\
\hline 0.50 & $20.93 \pm 0.09$ & $1.13 \pm 0.10$ & $0.75 \pm 0.12$ & $1.88 \pm 0.11$ \\
1.00 & $20.18 \pm 0.09$ & $1.07 \pm 0.10$ & $0.71 \pm 0.12$ & $1.78 \pm 0.11$ \\
1.51 & $19.91 \pm 0.09$ & $1.01 \pm 0.10$ & $0.71 \pm 0.12$ & $1.72 \pm 0.11$ \\
2.02 & $19.78 \pm 0.09$ & $1.02 \pm 0.10$ & $0.68 \pm 0.12$ & $1.69 \pm 0.11$ \\
& ${\text { Te }+16^{b}}^{\text {Active centaurs }}{ }^{c}$ & $0.97 \pm 0.02$ & $0.67 \pm 0.02$ & $1.64 \pm 0.03$ \\
Sun $^{d}$ & $0.85 \pm 0.16$ & $0.51 \pm 0.12$ & $1.32 \pm 0.27$ \\
& 0.64 & 0.35 & 0.99 \\
\hline
\end{tabular}

Notes. ${ }^{(a)}$ Aperture radius; ${ }^{(b)}$ colours of 523676 (2013 UL10) by Tegler et al. (2016); ${ }^{(c)}$ average colours for active centaurs, respectively. Literature data are taken from Jewitt $(2009,2015)$; Mazzotta Epifani et al. (2014); Peixinho et al. (2015). The value and the associated error have been computed considering the median (over 13 values) and the maximum error; ${ }^{(d)}$ solar colours in the modern Johnson-Cousins system (Holmberg et al. 2006).

excess due to comet-like activity around 523676 (2013 UL10) is clearly evident in Fig. 2, where for comparison we show also the stacked image of 2008 FC76, an inactive centaur observed during the same night. In order to better investigate the faint comet-like coma around the nucleus of 523676 (2013 UL10), we used a well-consolidated procedure that has been applied by Mazzotta Epifani et al. (2007, 2008), for instance, and was only slightly modified by Mazzotta Epifani et al. (2014). We compared four profiles of the target, centred on its optocentre in different directions, with a stellar profile derived from field stars in the same image (derived as an average of three central rows of the stellar trails perpendicular to the centaur proper motion), after normalisation to a value of 1 in the peak (the normalisation to 0 in the wings is ensured by sky subtraction). An example of the results for one of the single images in the field is shown in Fig. 3, where we also show the residuals of the centaur profile (with respect to an in-field stellar one).

In order to exclude any possible systematic in the night and to further confirm the presence of comet-like activity around this centaur, the same procedure was applied to the inactive centaur 2008 FC76, whose profile did not show any excess with respect to the stellar one. The residuals of 2008 FC76 are also shown in Fig. 3 for comparison.

\subsection{Colours}

Assuming that the nucleus of 523676 (2013 UL10) is a pointlike source embedded in a surrounding coma, we sampled the nucleus contribution (plus an unknown, but presumably small contribution from the near-nucleus coma) using the entire flux inside the photometric aperture corresponding to the stellar point spread function (PSF) of the image, that is, the average FWHM. The magnitude and colours of centaur 523676 (2013 UL10), derived in different optical apertures centred on the optocentre, are summarised in Table 3 . They agree well with the values found by Tegler et al. (2016), considering that these authors did not resolve the contribution of the (possibly present) dust coma.

Using a reference aperture of $D=1 \operatorname{arcsec}$ for the night of December $2015\left(\rho=0.50^{\prime \prime}\right)$, we derived the colours of the 523676 (2013 UL10) nucleus shown in Fig. 4, reported there together with those of inactive and active centaurs found in the literature (Jewitt 2009, 2015; Mazzotta Epifani et al. 2014; Peixinho et al. 2015; Tegler et al. 2016). To compile colours of active centaurs for which multiple values are reported relative to different apertures, we adopted the lowest tabulated value as 

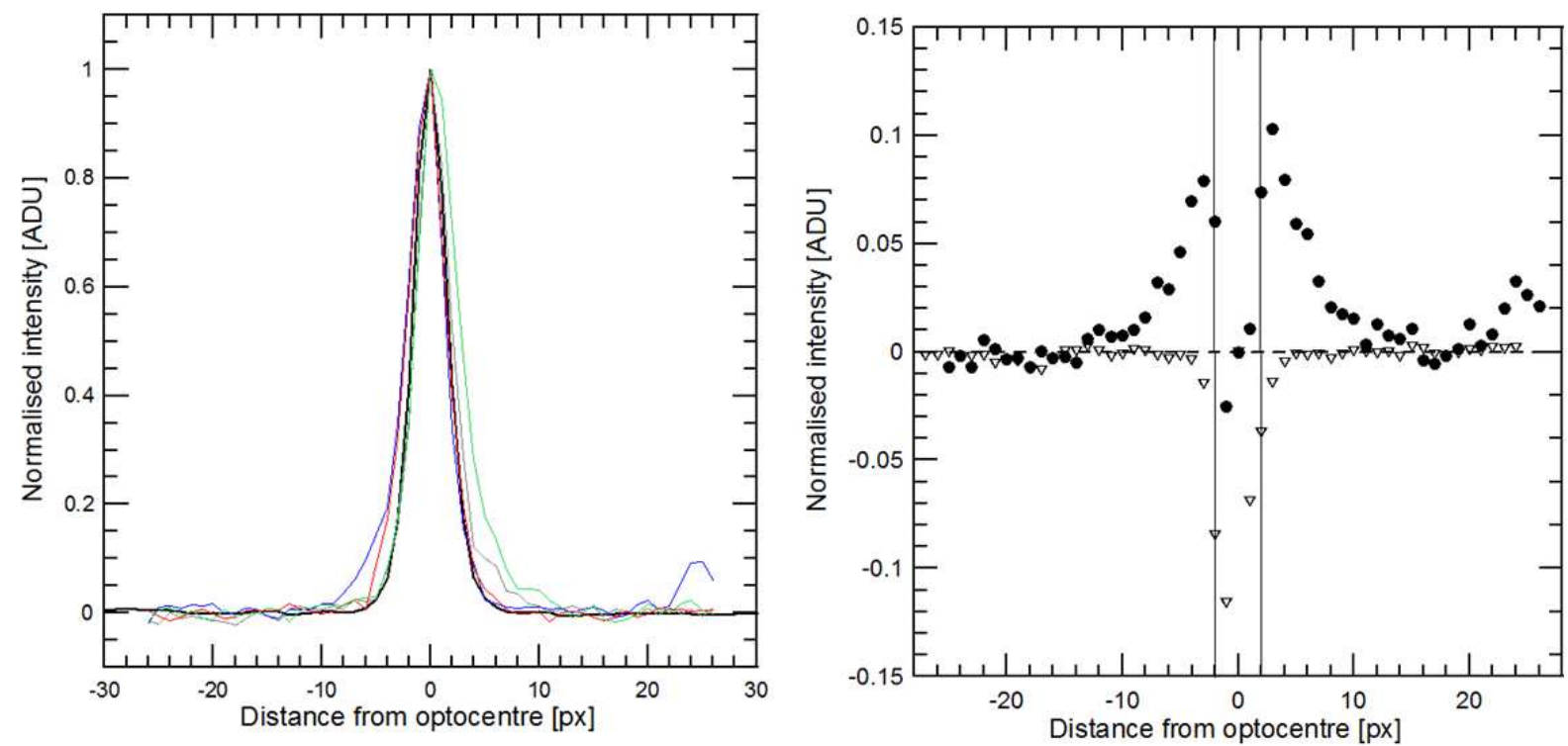

Fig. 3. Example of profile analysis for one $R$ image of 523676 (2013 UL10). Left panel: comparison of the four profiles in different directions (grey and blue lines: perpendicular and parallel to the apparent motion of the centaur, respectively; red and green lines: two orthogonal directions oriented at $45^{\circ}$ with respect to the two above) around the centaur's optocentre with the average stellar profile (thick black line). Right panel: comparison of residuals of centaur 523676 (2013 UL10) (filled dots) and 2008 FC76 (empty triangles) with respect to an in-field stellar profile.

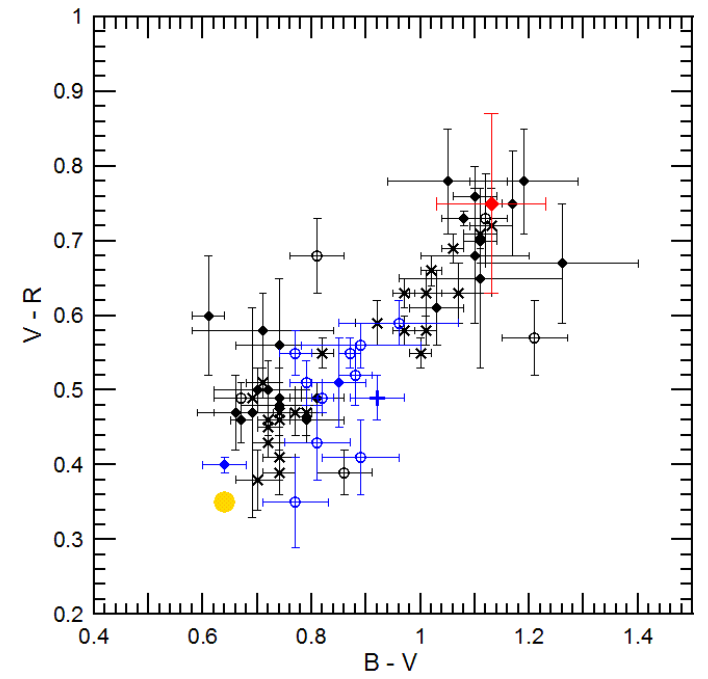

Fig. 4. Colour-colour diagram comparing the inactive centaurs (black symbols) with the active ones (blue symbols): literature data are taken from Peixinho et al. (2015; black and blue diamonds), Tegler et al. (2016; black asterisks), Jewitt (2009; blue open circles), Jewitt (2015; black and blue open circles), Mazzotta Epifani et al. (2014; blue cross). The yellow dot shows the colour of the Sun. The red diamond represents centaur 523676 (2013 UL10) (this work).

the nucleus colour to minimise the contribution of the dust coma surrounding the nucleus.

A well-defined gap among two clumps is not immediately evident; there are relatively few objects around $[B-R] \sim$ 1.50. Nevertheless, 523676 (2013 UL10), considering its nucleus colour (1.88 \pm 0.11 , see Table 3$)$ and taking into account its error bars, is within the limits of the traditional red group.

\subsection{Nucleus size}

As detailed above, the flux inside the photometric aperture corresponding to the average FWHM can be considered representative of the nucleus contribution (plus a small contribution from the surrounding coma). The $R$ magnitude in this region can therefore be used to estimate the upper limit to the geometric cross-section of the centaur nucleus, which, as extensively discussed in several papers (Lowry et al. 2003; Lowry \& Weissman 2005; Lowry \& Fitzsimmons 2005; Snodgrass et al. 2005, 2006; Mazzotta Epifani et al. 2007, 2008) for snapshot observations similar to those presented in this work, gives an excellent estimate of the (upper limit to the) effective radius of the sphere equivalent to the target. Following the formulation by Jewitt (1991), for a spherical object it is $A r_{N}^{2}=2.24 \times 10^{22} r^{2} \Delta^{2} 10^{0.4\left(m_{\text {SUN }}-m_{\text {nucleus }}+\alpha \beta\right)}$, where $A$ is the geometric albedo, $r_{N}$ is the radius of the body (expressed in meters), $m_{\mathrm{SUN}}$ the magnitude of the Sun in the same wavelength band of the body, $r$ and $\Delta$ are the heliocentric and geocentric distance of the target (expressed in au), respectively, and $\alpha$ and $\beta$ are the phase angle $\left(^{\circ}\right)$ and the phase coefficient $\left(\mathrm{mag} \mathrm{deg}^{-1}\right)$ in the phase-darkening function $\Phi(\alpha)=10^{-0.4 \alpha \beta}$, respectively.

Following Peixinho et al. (2004), we adopted $\beta=$ $0.11 \mathrm{mag} \mathrm{deg}^{-1}$. For the albedo, a wide spread of values has been measured within the centaur group: from 2.5 to $26 \%$, with a mean value of $6.9 \pm 3.9 \%$ (Duffard et al. 2014). Recent works (Fraser et al. 2014; Lacerda et al. 2014) investigated the question of a dependance of albedo on object colour: in particular, it has been shown that in a sample of 20 inactive centaurs divided into two clumps by the spectral slope $S^{\prime}$ at $S^{\prime}=25$ (in \% per $1000 \AA$ ), redder objects ( 8 in the whole sample) have a higher albedo value $A=0.12$ than the bluer ones $(A=0.06)$. This tendency of red objects to have higher albedo is also found in hot $\left(i \geq 5^{\circ}, r \geq\right.$ $30 \mathrm{au}$ ) trans-Neptunian objects (TNOs) with absolute magnitude $5 \leq H \leq 10$, even if the dispersion of albedo values is quite high. Adopting $A=0.12$ for centaur 523676 (2013 UL10), we obtain $D \leq 10 \mathrm{~km}$, quite a small size when compared to the average size of inactive centaurs, which is more than one order of magnitude larger (Bauer et al. 2003; Stansberry et al. 2008; Jewitt 2009; Perna et al. 2010). This is consistent with the fact that in general, active centaurs are found to be smaller than inactive ones (Mazzotta Epifani et al. 2017). 


\subsection{Dust production rate}

The comet-like activity around the nucleus shown by centaur 523676 (2013 UL10) during our observation was evident, even if quite weak. In order to obtain a first-order estimate of the dust production rate, we applied the so-called photometric method, derived from the method used to compute the dust production rate of active centaurs in the region between 5 and 12 au (Jewitt 2009). The image obtained in the $R$ filter was used as input in the model, as this filter is the less affected by possible gas contamination within the coma. We expect little contamination at such large heliocentric distances, given also the nondetection of $\mathrm{CO}$ rotational lines in the very few centaurs investigated, for example, which implies a strong depletion of exposed supervolatile ices on the nucleus surface (Jewitt et al. 2008).

We adapted and already applied this method to several active small bodies at different heliocentric distances and in particular to other active centaurs (see e.g. Mazzotta Epifani et al. 2011, 2014, 2017). We used the obtained photometric results (magnitude in the $R$ filter) in order to derive the cross-section $C_{\mathrm{d}}$ of the dust coma and the dust mass $M_{\mathrm{d}}$ of the coma itself, and we estimated the dust production rate $Q_{\mathrm{d}}$ taking into account the time of residence of grains in a defined portion of the projected coma. The model is a function of mainly (i) the size distribution of dust grains present in the coma $f(a)=a^{q}$, including also the limits ( $a_{-}$and $a_{+}$) of grains to be considered in the model; and (ii) the dust outflow velocity $v$. The latter is in turn strictly dependent not only on the heliocentric distance of the nucleus, which affects the gas production rate in a very complicated way, but also on the grain size, which affects the efficiency of dust coupling with the same gas.

For the power-law dust size distribution, we adopted a value of $q=-3.5$ for compact $\left(\rho=1000 \mathrm{~kg} \mathrm{~m}^{-3}\right)$ grains between $a_{-}=$ $1 \mu \mathrm{m}$ and $a_{+}=1 \mathrm{~mm}$, which results in an average grain scatterer of size $a=\sim 32 \mu \mathrm{m}$. This can be considered a realistic assumption supported by numerical models of dust tails for some comets (see e.g. a summary in Fulle 1999) and is here also used to be consistent to similar estimates of dust mass-loss rate for active centaurs in the past. We observed that the model results are very sensitive to the adopted average grain scatterer size.

For the dust outflow velocity, we recall that Probstein's theory (Probstein 1969) predicts that dust emitted by a spherical nucleus travels at $\sim 10 \%$ of the gas velocity, which for comet $\mathrm{C} / 1995 \mathrm{O} 1$ (Hale-Bopp) has been modelled to be $v(r)=v_{0}$. $\left(r_{0} / r\right)^{1 / 4}$, with $v_{0}=550 \mathrm{~m} \mathrm{~s}^{-1}$ and $r_{0}=5$ au (Biver 2002). Dust grains are not perfectly coupled with gas (depending on their size), and moreover, they likely do not travel radially across the projected coma section, therefore it is wise to take into account a range of grain velocities instead of a single value.

Figure 5 shows the output of the photometric model applied to 523676 (2013 UL10) for different values of the average grain scatterer, when adopting a grain outflow velocity from 1 to $100 \mathrm{~m} \mathrm{~s}^{-1}$, and a phase function applied with the Schleicher method (Schleicher et al. 1998; Schleicher 2007), which is based on a (slightly shallower) phase function tabulated for small phase angles ${ }^{3}$ and typical for dust (cometary) comae. The dependance of outflow velocity on grain size within comet-like comae is not straightforward, even if it is intuitive that in general, smaller grains travel faster than larger ones. Dots in Fig. 5 show results of the model when it is applied with the photometry derived for 523676 (2013 UL10) and three particular combinations of $a$ and $v$ that were modelled at 6 au for comet C/1995 O1 (HaleBopp; Weiler et al. 2003). For 523676 (2013 UL10), making the

3 asteroid.lowell.edu/comet/dustphaseHM_table.txt

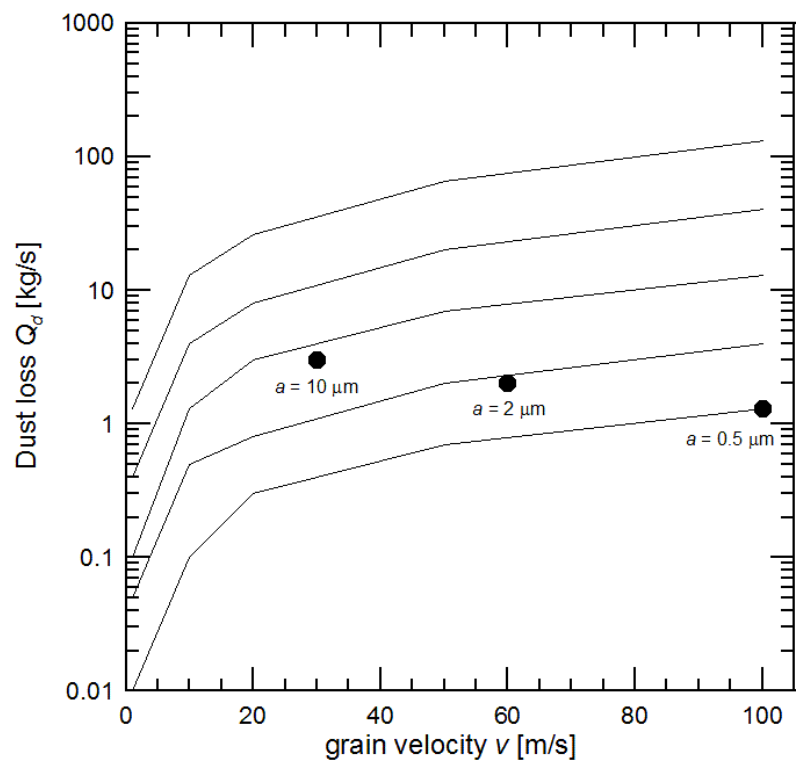

Fig. 5. Estimate of dust production rate $Q_{\mathrm{d}}$ for centaur 523676 (2013 UL10) for an average grain scatterer of size $a=1,3,10,32$, and $100 \mu \mathrm{m}$, respectively (continuous lines, from bottom to top). The filled dots represent values of $Q_{\mathrm{d}}$ obtained with the photometric model, applied for three particular combinations of $a$ and $v$ that were derived for comet C/1995 O1 (Hale-Bopp) at 6 au (see text).

assumption of $a=32 \mu \mathrm{m}$ and $v=20 \mathrm{~m} \mathrm{~s}^{-1}$, we obtain $Q_{\mathrm{d}} \sim$ $10 \mathrm{~kg} \mathrm{~s}^{-1}$ at $6.62 \mathrm{au}$ (shortly after perihelion passage). As said before, this should be regarded as a crude order of magnitude of the perihelion $Q_{\mathrm{d}}$, which is strongly model dependent: for instance, for $a=3 \mu \mathrm{m}$ and $v=50 \mathrm{~m} \mathrm{~s}^{-1}$, we obtain $Q_{\mathrm{d}} \sim 2 \mathrm{~kg} \mathrm{~s}^{-1}$ at $6.62 \mathrm{au}$.

\section{Discussion and future perspectives}

The issue of the colour diversity of inactive centaurs is a still-unexplained feature of this peculiar class of bodies. Being considered as transient objects, on their way towards the inner part of the solar system while coming from the Kuiper belt, centaurs represent a crucial element in formation and evolution models of primordial objects, and their surface properties (mainly the colours) depend on the evolutionary or primordial interpretation of their differences. The different surface colours could reflect their different formation location (a primordial, temperature-induced composition gradient) or could be due to the combined effect of quite recent evolutionary processes. This issue is further complicated by the presence of cometary activity: more and more active centaurs in the class are being discovered, and it is not yet possible at present to formally conclude at the $3 \sigma$ level of confidence that the inactive and active centaurs have different colour distributions (Peixinho et al. 2015; Jewitt 2015).

The fallback blanketing, first discussed by Jewitt (2015), has been invoked as the primary cause of the bluishing of the centaur surface, as a consequence of the outgassing activity destroying the red matter that might be present on the nucleus, blanketing it by fallback debris composed of fresh, un-irradiated material expelled from below. Timescales $\tau_{B}$ for the blanketing process are very uncertain, and the issue is further complicated by the fact that comet-like activity in the centaurs is often episodic (see e.g. the peculiar case of centaur 174P/Echeclus; Choi et al. 2006) and highly dependent on their dynamical (i.e. semi-major axis and average distance from the Sun) and physical properties 


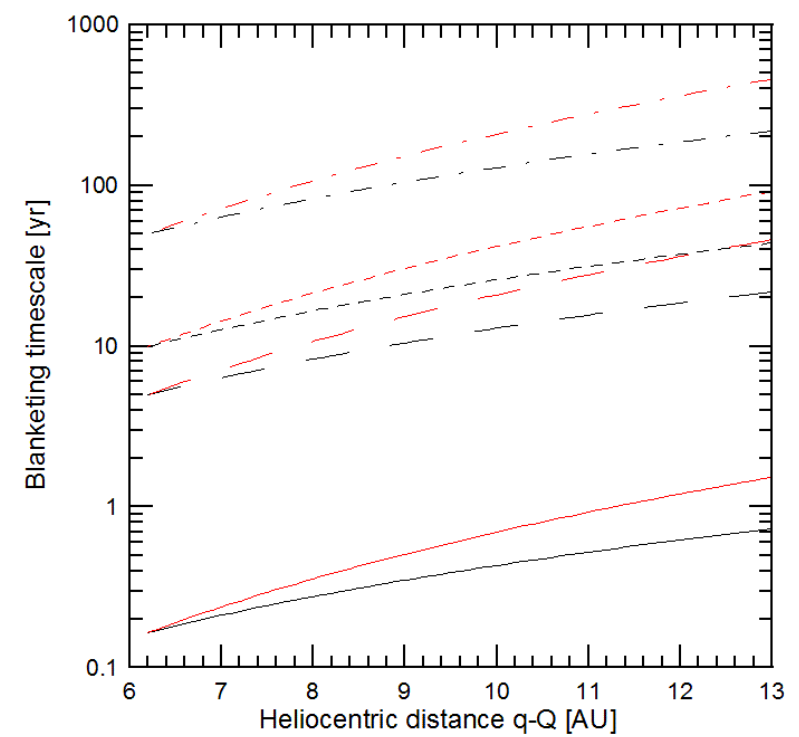

Fig. 6. Blanketing timescale estimates from 6.2 (time of observation, $\sim$ perihelion) to 13 au of 523676 (2013 UL10), assuming different combinations of parameters. Continuous lines: $Q_{\mathrm{d}}$ at perihelion $=10 \mathrm{~kg} \mathrm{~s}^{-1}$, $f_{\mathrm{B}}=0.06$; dashed lines: $Q_{\mathrm{d}}$ at perihelion $=10 \mathrm{~kg} \mathrm{~s}^{-1}, f_{\mathrm{B}}=0.001$; longdashed lines: $Q_{\mathrm{d}}$ at perihelion $=2 \mathrm{~kg} \mathrm{~s}^{-1}, f_{\mathrm{B}}=0.01$; dot-dashed lines: $Q_{\mathrm{d}}$ at perihelion $=2 \mathrm{~kg} \mathrm{~s}^{-1}, f_{\mathrm{B}}=0.001$. Black lines are for a $Q_{\mathrm{d}}$ drop of $r^{-2}$, red lines are for a $Q_{\mathrm{d}}$ drop of $r^{-3}$. See text for more details.

(e.g. nucleus size and active surface fraction). To obtain an estimate of expected $\tau_{B}$ for 523676 (2013 UL10), we used an approach similar to that reported by Jewitt (2015; their Sect. 4.3), estimating the time needed to blanket a spherical nucleus of radius $r_{N}=5 \mathrm{~km}$ to a depth of $\Delta l=10 \mu \mathrm{m}$. Two different values of $Q_{\mathrm{d}}$ at perihelion were considered, as obtained for two different combinations of dust size and velocity (see above, Sect. 3.4). The fraction $f_{\mathrm{B}}$ of ejecta that falls back to the nucleus surface and can therefore form a layer capable of hiding the surface material is quite difficult to estimate, since it is strictly dependent on nucleus shape and dynamical properties (such as rotation), uniformity of emission, dust properties (e.g. size distribution), and so on. Therefore, we adopted a range of plausible values for $f_{\mathrm{B}}$ from 0.001 to 0.06 to compute $\tau_{B}$ at perihelion, taking into account values estimated by Jewitt (2002) for fast grains and rescaling them to slow grains. We then took into account the increase in the blanketing timescales along the centaur orbit due to the increase in centaur heliocentric distance: Jewitt (2009) concluded that the mass-loss rate in active centaurs can drop faster than $r^{-2}$ if $Q_{\mathrm{d}}$ is not driven simply by the sublimation of an exposed (super)volatile.

Results are shown in Fig. 6: taking into account all the caveats discussed above on comet-like activity estimates (uncertainties on dust parameters, episodic activity, not uniform emission, and blanketing of the nucleus surface), we can reasonably assume that $\tau_{B}$ is of the order of fractions to several tens of years.

Within this framework, it is clear that the probability of observing outgassing activity on red surface in centaurs (resurfacing in action, as it would be for 523676 (2013 UL10)) is very low: assuming an average $\tau_{B}=10 \mathrm{yr}$ and an average centaur lifetime $\mathcal{L}=10^{7} \mathrm{yr}$, the chance of observing 523676 (2013 UL10) in the above conditions is $\tau_{B} / \mathcal{L}=10^{-6}$, making the case of observation presented in this work very fortuitous. Incidentally, we note that our observation of only one red active centaur within the whole sample of red centaurs cannot be regarded without taking into account that not all centaurs have been equally searched for activity.

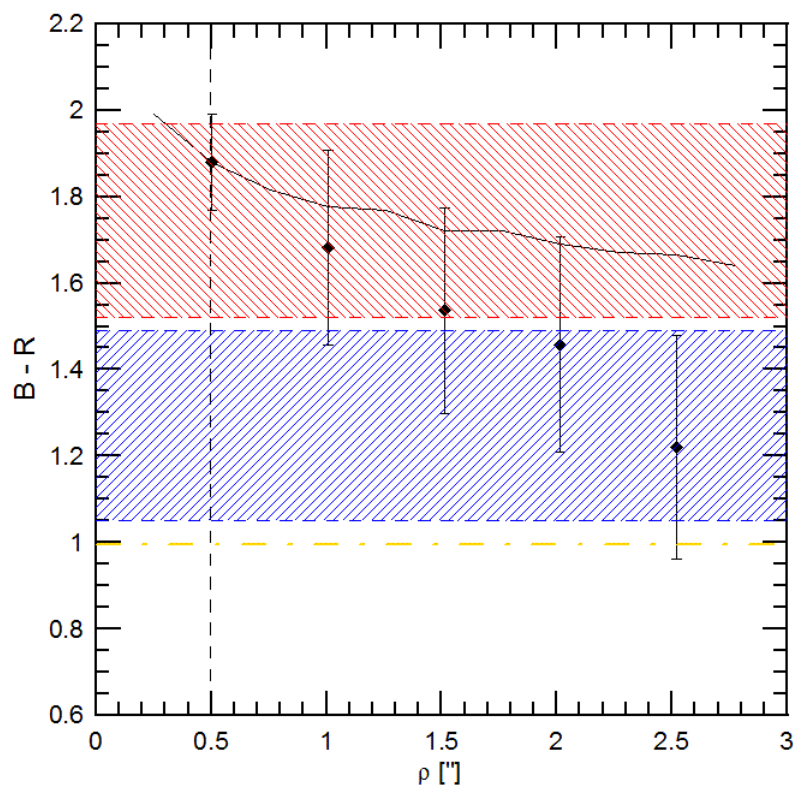

Fig. 7. Comparison of integrated $[B-R]$ colour of centaur 523676 (2013 UL10) (continuous black line) with respect to the colour derived in concentric annular regions with increasing distance from the optocentre (dots). Each dot represent the $[B-R]$ colour derived in the annulus between that $\rho$ value and the preceding one. The blue and red dashed areas indicate lower and upper limits of the $[B-R]$ colours of the blue and red inactive centaur groups, respectively, when adopting a gap at $[B-R]=1.50$. The yellow dot-dashed line indicates the $[B-R]$ colour of the Sun. The vertical dashed line indicates the considered limit for the nucleus (FWHM).

On the other hand, the fact that the resurfacing process is just started for 523676 (2013 UL10) seems to be supported by the significant difference in the colours of nucleus and surrounding dust, with the latter being more neutral than the underlying nucleus. Figure 7, where the integrated $[B-R]$ colour of 523676 (2013 UL10) is reported together with the colour derived in concentric anular regions with increasing distance from the optocentre, shows that this appears to be the case for our target: the dust colour in outer coma regions is consistent with neutral clump colours within data uncertainty.

We then point out that the blanketing model we used is probably too simple and should be revised and further constrained, as some alternative assumptions may be considered (e.g. a most likely sporadic activity instead of continuous activity). As said before, the blanketing physics is strictly dependent on the dynamical and physical properties of the centaur and on their evolution along the orbit.

For the reasons above, it is crucial to regularly monitor the surface colours of this centaur within the next few years in order to verify the evolution of its surface colours also in combination with the level of persistency of the comet-like activity of the centaur, which currently is on its outbound orbital branch. Incidentally, we recall that Kanuchova et al. (2012) showed by means of dedicated laboratory experiments that a combination of space weathering processes and competing resurfacing agents can continuously modify the surface colours of small bodies in the outer solar system, which can move on a colour-colour diagram (from blue to red colours and vice versa) on timescales much shorter than typical $\mathcal{L}$ for centaurs.

In a more general framework, we also remark on the strong bias against small objects in the current colour dataset that is available for centaurs, since they are difficult to observe without large telescopes. Active centaurs tend to be smaller than inactive 
ones, but in smaller objects, the comet-like activity eventually shown for a short time could remain unresolved without deep observations and long-term monitoring. This could also explain why they are flagged as inactive and placed in the red clump, for example.

Acknowledgements. D.P. has received funding from the European Union's Horizon 2020 research and innovation programme under the Marie SklodowskaCurie grant agreement no. 664931.

\section{References}

Bauer, J. M., Meech, K. J., Fernandez, Y. R., et al. 2003, Icarus, 166, 195 Biver, N., 2002, Earth Moon Planets, 90, 5

Capria, M. T., Coradini, A., De Sanctis M. C., et al. 2000a, A\&A, 357, 359

Capria, M. T., Coradini, A., De Sanctis M. C., et al. 2000b, AJ, 119, 3112

Choi, Y.-J., Weissman, P. R., Chesley, S., et al. 2006, CBET, 563, 1

Dotto, E., Barucci, M. A., \& de Bergh, C. 2003, EM\&P, 92, 157

Duffard, R., Pinilla-Alonso, N., Santos-Sanz, P., et al. 2014, A\&A, 564, A92

Fraser, W. C., \& Brown, M. E. 2012, ApJ, 749, 33

Fraser, W. C., Brown, M. E., Morbidelli, A., et al. 2014, ApJ, 782, 100

Fulle, M. 1999, Planet. Space Sci., 47, 827

Hainaut, O. R., \& Delsanti, A. C. 2002, A\&A, 389, 641

Holmberg, J., Flynn, C., \& Portinari, L. 2006, MNRAS, 367, 449

Horner, J., Evans, N. W., Bailey M. E., et al. 2004, MNRAS, 354, 798

Jewitt, D. 1991, in Comets in the Post-Halley Era, ed. 1, eds. R. L. Newburn,

M. Jr. Neugebauer, \& J. Rahe (Kluwer Academic Publisher)

Jewitt, D. 2002, AJ, 123, 1039

Jewitt, D. 2009, AJ, 137, 4296

Jewitt, D. 2015, AJ, 150, 6, 201

Jewitt, D., Garland, C. A., \& Aussel, H. 2008, AJ, 135, 400

Kanuchova, Z., Brunetto, R., Melita, M., \& Strazzulla, G. 2012, Icarus, 221, 12

Kulyk, I., Korsun, P., Rousselot, P., et al. 2016, Icarus, 271, 314

Lacerda, P. 2013, MNRAS, 428, 1818

Lacerda, P., Fornasier, S., Lellouch, E., et al. 2014, ApJ, 793, L2

Landolt, A. U. 1992, AJ, 104, 340
Levison, H. F., \& Duncan, M. J. 1997, Icarus, 127, 13

Lowry, S. C., \& Fitzsimmons, A. 2005, MNRAS, 358, 641

Lowry, S. C., \& Weissman, P-R. 2005, Icarus, 164, 492

Lowry, S. C., Fitzsimmons, A., \& Collander-Brown, S. 2003, A\&A, 397, 329

Luu, J. X., \& Jewitt, D. C. 1990, AJ, 100, 913

Mazzotta, Epifani, E., Palumbo, P., Capria, M. T., et al. 2006, A\&A, 460, 935

Mazzotta, Epifani, E., Palumbo, P., Capria, M. T., et al. 2007, MNRAS, 381, 713

Mazzotta, Epifani, E., Palumbo, P., Capria, M. T., et al. 2008, MNRAS, 390, 265

Mazzotta Epifani, E., Dall'Ora M., Perna, D., et al. 2011, MNRAS, 415, 3097

Mazzotta Epifani, E., Perna, D., Licandro, J., et al. 2014, A\&A, 565, A69

Mazzotta Epifani, E., Perna, D., Dotto, E., et al. 2017, A\&A, 597, A59

Meech, K. J., \& Belton M. J. S. 1990, AJ, 100, 1323

Meech, K. J., \& Svoren, J. 2004, in Comets II, eds. M.C. Festou, H.U. Keller, \& H. A. Weaver (Tucson, AZ: University Arizona Press)

Melita, M. D., \& Licandro, J. 2012, A\&A, 539, A144

Notesco, G., Bar-Nun, A., \& Owen, T. 2003, Icarus, 162, 183

Peixinho, N., Doressoundiram, A., Delsanti, A., et al. 2003, A\&A, 410, L29

Peixinho, N., Bohenhardt, H., Belskaya, I., et al. 2004, Icarus, 170, 153

Peixinho, N., Delsanti, A., Guilbert-Lepoutre, A., et al. 2012, A\&A, 546, A86

Peixinho, N., Delsanti, A., \& Doressoundiram, A. 2015, A\&A, 577, A35

Perna, D., Barucci, M. A., Fornasier, S., et al. 2010, A\&A, 510, A53

Perna, D., Dotto, E., Barucci, M. A., et al. 2013, A\&A, 554, A49

Prialnik, D. 1997, ApJ, 478, 547

Probstein, R. F. 1969, in Problems of Hydrodynamics and Continuum Mechanics, eds. Bishop, et al. (Philadelphia, PA: Society for Industrial and Applied Mathematics), 568

Schleicher, D. G. 2007, Icarus, 190, 406

Schleicher, D. G., Millis, R. L., \& Birch, P. V. 1998, Icarus, 132, 397

Snodgrass, C., Fitzsimmons, A., \& Lowry, S. C. 2005, A\&A, 444, 287

Snodgrass, C., Lowry, S. C, \& Fitzsimmons, A. 2006, MNRAS, 373, 1590

Stansberry, J., Grundy, W., Brown, M., et al. 2008, in The Solar System Beyond Neptune, eds. M.A. Barucci, H. Boehnhardt, D.P. Cruikshank, \& A. Morbidelli (Tucson, AZ: University Arizona Press)

Tegler, S. C., \& Romanishin, W. 2003, Icarus, 161, 181

Tegler, S. C., Romanishin, W., \& Consolmagno G. 2016, AJ, 152, 210

Tiscareno, M. S., \& Malhotra R. 2003, AJ, 126, 3122

Weiler, M., Rauer, H., Knollenberg J., Jorda L., \& Helbert J. 2003, A\&A, 403, 313 